Table 1 Summary of laboratory findings

\begin{tabular}{llll}
\hline Test & $\begin{array}{l}\text { Values on } \\
\text { admission }\end{array}$ & $\begin{array}{l}\text { Values 12 hours } \\
\text { after admission }\end{array}$ & $\begin{array}{l}\text { Reference range } \\
\text { (SI units) }\end{array}$ \\
\hline WBC & 18.9 & 29.0 & $4.8-10.8 \times 10^{9} / 1$ \\
Haemoglobin & 10.1 & 9.5 & $14-17 \mathrm{~g} / \mathrm{dl}$ \\
Platelets & 238 & 175 & $150-450 \times 10^{9} / 1$ \\
Sodium & 133 & 135 & $134-144 \mathrm{mmol} / 1$ \\
AST & 101 & 342 & $8-37 \mathrm{U} / 1$ \\
ALT & 75 & 248 & $0-39 \mathrm{U} / 1$ \\
Potassium & 4.6 & 4.7 & $3.7-4.9 \mathrm{mmol} / 1$ \\
Creatinine & 105 & 106 & $53-106 \mu \mathrm{mol} / 1$ \\
Blood urea nitrogen & 8.6 & 8.9 & $2.9-8.2 \mathrm{mmol} / 1$ \\
Total bilirubin & 46.2 & 42.8 & $2-21 \mu \mathrm{mol} / 1$ \\
LDH & 1118 & 1215 & $102-228 \mathrm{U} / 1$ \\
CK & 1121 & 660 & $0-183 \mathrm{U} / 1$ \\
Troponin-T & 5.5 & 5.4 & $0-0.1 \mathrm{ng} / 1$ \\
\hline
\end{tabular}

WBC = white blood cells; AST = aspartate aminotransferase; ALT = alanine aminotransferase; $\mathrm{LDH}=$ lactate dehydrogenase.

renal disease. ${ }^{34}$ Ours is the first report to indicate that such spurious rises may also be seen in the presence of intact renal function.

As there were no clinical electrocardiograph tracings in our patient suggestive of myocardial infarction, we believe that the increased concentrations of troponin- $T$ were an erroneous indicator of myocardial infarction. There are two possible reasons for the rise in troponin- $T$ concentrations: firstly, as a result of the existence of minor myocardial injuries undetectable in the ECG. ${ }^{1}$ Lack of an increase in the CK-MB fraction could be explained by the fact that cardiac troponin- $T$ is known to have a higher sensitivity and therefore a lower diagnostic efficiency than $\mathrm{CK}-\mathrm{MB}$ in the diagnosis of myocardial infarction. ${ }^{1}$ A second, alternative explanation, and one that we favour, is that the raised cardiac troponin- $T$ concentration is a consequence of the multiple disorders, septicaemia and widespread malignancy present in our patient. There is unpublished information from retrospective reviews of medical records to indicate that patients positive for troponin- $T$ and negative for
CK-MB have multiple illnesses. As the presence of multiple illnesses is often a complication of and not an uncommon clinical phenomenon in widespread malignant disease, we believe that this anecdotal information may be of use. It is possible that follow up values after the acute period may have provided an additional insight into the cause of the increases observed in the present patient. However, this information was not available as the patient died two days after admission to hospital. Permission to perform a necropsy was not granted and the cause of death was presumed to be overwhelming sepsis.

The CK-BB isoenzyme has been proposed as a tumour marker as it indicates the presence of solid organ malignancies. ${ }^{5}$ There is also an isolated case report of a patient with MDS with an elevated CK-BB fraction. ${ }^{6}$ Thus, the increased CK-BB fraction observed in our patient may be attributable to either or all of the three malignancies.

Increases in cardiac troponin- $T$ concentrations in the absence of diagnostic ECG changes and an increase in the CK-MB fraction support the exclusion of myocardial infarction as a clinical possibility and re-affirm the use of CK-MB as a diagnostic tool in this condition.

1 Bhayana V, Henderson AR. Biochemical markers of myocardial damage. Clin Biochem 1995;28:1-29.

2 Kobayashi S, Tanaka M, Tamura N, Hashimoto H, Hirose S. Serum cardiac troponin $\mathrm{T}$ in polymyositis/dermatomyositis. Lancet 1992;340:726.

3 Wu AH, Valdes R Jr, Apple FS, Gornet T, Stone MA Mayfield-Stokes S, et al. Cardiac troponin-T immunoassay for diagnosis of acute myocardial infarction. Clin Chem 1994;40:900-7.

4 Croitoru M, Taegtmeyer $H$. Spurious rises in troponin- $T$ in end-stage renal disease. Lancet 1995;346:974.

5 Abbott B, Lott JA. Reactivation of serum creatine kinase isoenzyme $\mathrm{BB}$ in patients with malignancies. Clin Chem 1984;30: $1861-3$

6 Crook M, Williams A, Sankarlingam A, Tutt P. Raised concentration of plasma creatine kinase $B B$ isoenzyme in myelodysplasia. f Clin Pathol 1994;47:552-3.

\title{
High temperature antigen retrieval and loss of nuclear morphology: a comparison of microwave and autoclave techniques
}

Immunocytochemistry and Molecular

Pathology Unit, Department of Pathology, University Hospital of Wales, Heath Park, Cardiff CF4 4XN

Correspondence to: Dr NCA Hunt, Clinical Lecturer Nuffield Department of Pathology and Microbiology, John Radcliffe Hospital, Headington,

Oxford OX3 9DU.

Accepted for publication 4 June 1996

\section{N C A Hunt, R Attanoos, B Jasani}

\begin{abstract}
The use of high temperature antigen retrieval methods has been of major importance in increasing the diagnostic utility of immunocytochemistry. However, these techniques are not without their problems and in this report attention is drawn to a loss of nuclear morphological detail, including mitotic figures, following microwave antigen retrieval. This
\end{abstract}

was not seen with an equivalent autoclave technique. This phenomenon was quantified using image analysis in a group of $B$ cell lymphomas stained with the antibody L26. Loss of nuclear morphological detail may lead to difficulty in identifying cells accurately, which is important in the diagnostic setting-for example, when trying to distinguish a malignant lymphoid infiltrate within a mixed cell popu- 
lation. In such cases it would clearly be wise to consider the use of alternative high temperature retrieval methods and accept their slightly lower staining enhancement capability compared with the microwave technique.

(F Clin Pathol 1996;49:767-770)

Keywords: antigen retrieval, artefacts, immunocytochemistry.

The masking of tissue antigens in routinely processed histological specimens has been a major obstacle to the application of immunocytochemistry to archival material. A variety of techniques have been successfully developed to reveal such antigens, including enzymatic and high temperature methods. ${ }^{1}$ These techniques have been important in overcoming the obvious limitations imposed by the dependence on frozen tissue and their development has permitted the diagnostic utility of immunocytochemistry to increase dramatically. However, these methods are not without disadvantages. Here, we present histomorphometric data from a study comparing our standard autoclave and microwave pretreatment protocols in a group of B cell lymphomas stained with the L26 antibody (anti-CD20 B cell lineage marker). Our results demonstrate a potentially significant difference between these methods with implications for diagnostic work.

\section{Methods}

Six cases of non-Hodgkin's B cell lymphoma were identified retrospectively from the archives of the Department of Histopathology, University Hospital of Wales. All cases were of diffuse, centroblastic/centrocytic type. Sections, 4-5 $\mu \mathrm{m}$ thick, were cut from each tumour and were untreated or pretreated in an autoclave ( $0.01 \mathrm{M}$ sodium citrate buffer, $\mathrm{pH}$ $6.5 ; 15$ minutes at $\left.120^{\circ} \mathrm{C} ; 15 \mathrm{psi}\right),{ }^{2}$ or pretreated in a microwave oven $(0.01 \mathrm{M}$ sodium citrate buffer, $\mathrm{pH} 6.5 ; 15$ minutes at $800 \mathrm{~W}$ (Matsui TC, Harrow, UK)). ${ }^{2}$

Following pretreament, all sections were stained with L26 (Dako, High Wycombe, UK) (diluted 1 in 40 ) and incubated at $4^{\circ} \mathrm{C}$ overnight. Sections were then stained using a simple, indirect immunoperoxidase method. All sections then underwent histomorphometric analysis of mean nuclear area and mitotic index. All samples were examined blind.

To assess the apparent difference in nuclear size observed between the control, autoclave and microwave treated sections, the cross-

Table 1 Comparison of mean nuclear area of centrocytic/centroblastic cells and mitotic index with and without pretreatment by autoclave or microwave antigen retrieval methods. Results expressed as mean (SD) for area

\begin{tabular}{|c|c|c|c|c|c|c|}
\hline \multirow[b]{2}{*}{$\begin{array}{l}\text { Case } \\
\text { number }\end{array}$} & \multicolumn{2}{|l|}{ Control } & \multicolumn{2}{|l|}{ Autoclave } & \multicolumn{2}{|l|}{ Microwave } \\
\hline & Area $\left(\mu m^{2}\right)$ & $\begin{array}{l}\text { Mitotic } \\
\text { count }\end{array}$ & Area $\left(\mu m^{2}\right)$ & $\begin{array}{l}\text { Mitotic } \\
\text { count }\end{array}$ & Area $\left(\mu m^{2}\right)$ & $\begin{array}{l}\text { Mitotic } \\
\text { count }\end{array}$ \\
\hline 1 & $19.03(4.42)$ & 11 & $20.87(5.75)$ & 18 & $32.05(8.70)$ & 4 \\
\hline 2 & $18.25(3.29)$ & 8 & $17.22(4.46)$ & 8 & 36.17 (13.29) & 3 \\
\hline 3 & $20.56(4.65)$ & 30 & $23.24(5.08)$ & 44 & $31.81(5.89)$ & 7 \\
\hline 4 & $17.29(3.49)$ & 37 & $16.43(2.44)$ & 51 & $30.55(6.57)$ & 17 \\
\hline 5 & 16.43 & 33 & $18.22(4.38)$ & 46 & $31.97(5.69)$ & 17 \\
\hline 6 & $17.36(3.65)$ & 17 & $20.72(4.37)$ & 15 & $30.12(6.49)$ & 2 \\
\hline
\end{tabular}

sectional area of 300 randomly selected nuclei was measured from each section using an IBAS image analyser (Kontron, Ecking, Germany), with a Videoplan software package, coupled to an Olympus (London, UK) BH2 microscope. The data were gathered by interactively defining the nuclear circumference using computer generated graphics superimposed over a live, colour television image. From these data the mean cross-sectional nuclear area for each section was calculated. Mitotic indices were then determined for each section using a slight modification of the methodology described by van Diest et al. ${ }^{3}$ In each case 50 high power fields were counted using an Olympus $\mathrm{BH} 2$ \& microscope $(\times 40$ objective; field diameter 500 $\mu \mathrm{m})$.

The histomorphometric data were analysed by comparison of means using standard errors. The mitotic indices were analysed for significant differences using a Wilcoxon rank sum test for non-parametric data.

\section{Results}

The results of the histomorphometric analysis of nuclear area are presented in table 1 . There seems to be a constant difference between the sections pretreated with microwaves compared with the control and the autoclave pretreated sections in terms of nuclear area, which was consistently higher in the former. This reaches statistical significance in each case with the null hypothesis being rejected at $p<0.001$ (table 1). Analysis of the mitotic indices for sections from each case showed a consistent difference between the sections pretreated with microwaves compared with both control and autoclave pretreated sections. In all cases the mitotic index was lower in the microwave treated sections.

In qualitative terms a number of differences were noted between the variously treated sections. The microwave pretreated sections showed a clear increase in intensity of staining when compared with controls. The autoclave pretreated sections also showed an increase in staining intensity compared with the control sections, although overall enhancement was slightly less than in the microwave pretreated group. However, there was also a clear, qualitative difference between the sections in terms of cellular morphology with the control sections having the best morphology and those pretreated with microwaves the worst. The autoclave pretreated group had lost much less morphological detail than the microwave $\mathbb{D}$ pretreated group (fig 1) and compared favourably with the control group. Specifically, the cells in the microwave pretreated specimens appeared rather swollen with associated loss of nuclear detail and often complete blurring of mitotic figures. These observations are, we believe, the qualitative correlates of the quantitative data presented above.

\section{Discussion}

Immunocytochemistry has become an invaluable tool in routine diagnostic histopathology as well as an important research technique. The development of antigen retrieval tech- 

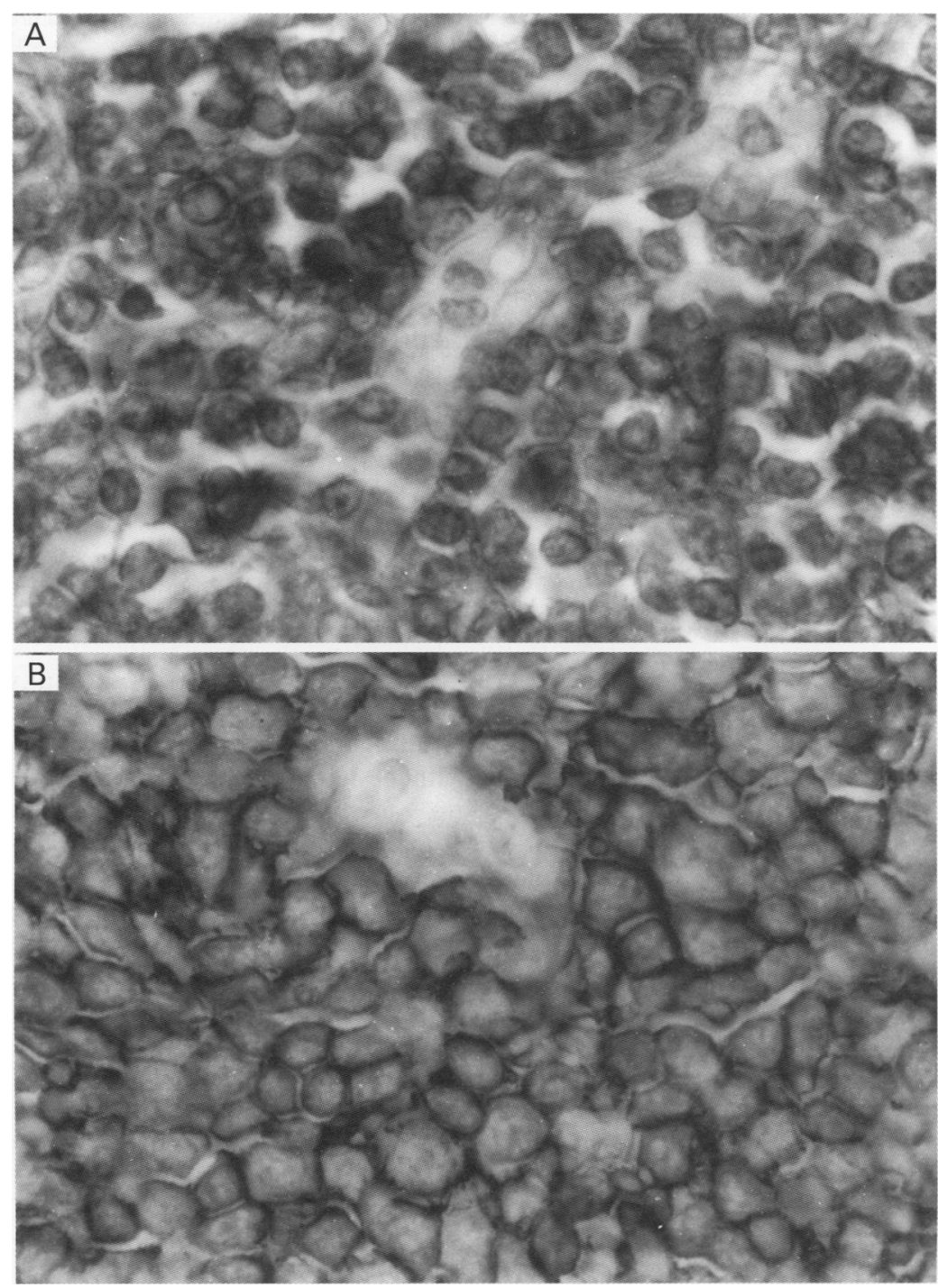

Figure 1 Photomicrographs showing (A) an autoclave pretreated section and $(B)$ a microwave pretreated section of lymphoma (original magnification $\times 200$ ).

niques has been instrumental in this process as it has permitted the application of antibodies to routinely processed tissue samples and has, in many cases, abrogated the need for frozen material. Such antigen retrieval can be achieved using both enzymatic and, more recently, high temperature techniques. ${ }^{1}$ The former techniques, whilst offering the potential for using formalin fixed, paraffin wax embedded material for immunocytochemistry, were difficult to standardise and do not offer significant staining enhancement with many antibodies. ${ }^{4}$ The latter techniques involve superheating tissue sections with either a microwave oven, ${ }^{5}$ autoclave $^{6}$ or pressure cooker. ${ }^{7}$ There are a number of possible mechanisms likely to be responsible for antigen retrieval using these techniques, including breakage of formaldehyde induced protein cross linkages, denaturation of proteins to reveal previously masked epitopes and unmasking of epitopes by removal of calcium ions. ${ }^{4}$ To date, most experience has been gained with microwave techniques, perhaps because workers already have experience with this technology. However, microwaves have certain drawbacks including the fact that they work best with a small number of cases, problems with evaporation of buffer, inconsistent staining due to local superheating, and their relatively frequent mechanical failures due to overuse. ${ }^{7}$ In addition, we have also noted in our own work on B cell lymphomas that microwave pretreatment seems to impair cellular morphology significantly. A diminution in morphology following microwave pretreatment has also been noted by other workers, ${ }^{5}$ although they do not seem to have assessed this phenomenon in any detail. Because of the implications of this in diagnostic work, especially when only limited material is available and enhancement is needed, we decided to undertake a formal study of this phenomenon as presented in this short report.

The cellular distortion with microwave pretreatment of tissue sections is reflected in the increase in mean nuclear area and the apparent decrease in the mitotic index. While it is possible to speculate on the underlying changes responsible for these observations, it would be rather more difficult to delineate these in exact detail. However, one possibility is that microwaves cause some structural damage to intracellular macromolecules which, in turn, leads to an increase in the number of osmotically active moieties within the nuclear compartment, thus attracting water and causing nuclear swelling. An intrinsic property of microwaves is proposed as superheating alone cannot be the answer as evinced by the much better morphological preservation using an autoclave. Such changes would certainly explain the increase in mean nuclear area observed and furthermore, this swelling would have the effect of blurring mitotic figures and making them less easy to distinguish accurately from apoptotic bodies or lymphocytes and thus less likely to be counted. Given that this study was undertaken using the optimised protocols used in our laboratory for diagnostic work, these observed changes may have important consequences for selection of retrieval methods in the diagnostic setting when it becomes important to identify accurately the cells which have been stained. Both mitotic figures and morphology may be of importance in achieving this-for example, when trying to distinguish a malignant lymphoid infiltrate within a mixed cell population. In such cases it would clearly be wise to consider the use of alternative high temperature retrieval methods and accept their slightly lower staining enhancement capability compared with the microwave technique.

1 Cattoreti G, Pileri S, Parravicini C, Becker MHG, Poggi S, Bifulco C, Key G, et al. Antigen unmasking on formalinfixed, paraffin-embedded tissue sections. $\mathcal{F}$ Pathol 1993, 171:83-98.

2 Banfalvi A, Navabi H, Bier B, Bocker W, Jasani B, Schmid K Wet autoclave pretreatment for antigen retrieval in diagnostic immunohistochemistry. F Pathol 1994;174:223-8.

3 van Diest PJ, Baak JPA, Matze-Cok P, Wisse-Brekelmans ECM, van Galen CM, Kurver PHJ, et al. Reproducibility of mitosis counting in 2469 breast cancer specimens: Results from the multicenter morphometric mammary
carcinoma project. Hum Pathol 1992;23:603-7.

4 Shi SR, Gu J, Kalra KI, Chen T, Cote RJ, Taylor CR. Antigen retrieval technique: A novel approach to immunocytochemistry on routinely processed tissue sections. Ciell Vision 1995;2:6-22.

5 Shi S-R, Key ME, Kalra KL. Antigen retrieval in formalinfixed, paraffin-embedded tissues: an enhancement method 
for immunohistochemical staining based on microwave oven heating of tissue sections. $f$ Histochem Cytochem 1991;39:741-8.

6 Navabi H, Douglas-Jones A, Bankfalvi A, Schmid K, Suzuki $\mathrm{K}$, Katoh $\mathrm{R}$, et al. Wet autoclave pretreatment: a reliable alternative to microwave technique for antigen retrieval [abstract]. $\mathscr{f}$ Pathol 1994;172:50A.
7 Norton AJ, Jordan S, Yeomans P. Brief, high-temperature heat denaturation (pressure cooking): a simple and effective method of antigen retrieval for routinely processed tissues. F Pathol 1994;173:371-9.

8 Morgan JM, Navabi H, Schmid K, Jasani B. Possible role of tissue-bound calcium ions in citrate-mediated hightemperature antigen retrieval. $\mathcal{f}$ Pathol 1994;174:301-7.

\author{
J H Shanks, S S Banerjee, B P Eyden
}

\title{
Focal rhabdomyosarcomatous differentiation in primary liposarcoma
}

\begin{abstract}
A unique case of primary myxoid liposarcoma of the thigh, in which focal pleomorphic areas were present containing rhabdomyoblasts, is described. Focal rhabdomyosarcoma in liposarcoma has only rarely been reported previously and only in dedifferentiated liposarcomas of the retroperitoneum. All but one have been recurrences with rhabdomyoblasts being absent in the primary liposarcoma. As rhabdomyoblasts were only focally present, the present case is regarded as liposarcoma with focal divergent rhabdomyoblastic differentiation rather than malignant mesenchymoma.

(F Clin Pathol 1996;49:770-772)
\end{abstract}

Keywords: liposarcoma, rhabdomyosarcoma, malignant mesenchymoma.

Liposarcoma may contain benign or malignant heterologous mesenchymal elements. Benign cartilage, ${ }^{1}$ smooth muscle ${ }^{2}$ or rarely bone ${ }^{1}$ have been described in well differentiated liposarcoma/atypical lipomatous tumours or in myxoid liposarcoma. Until now, malignant heterologous elements have been found only in dedifferentiated liposarcoma. ${ }^{13-5}$

Malignant myogenic elements found in liposarcoma have included leiomyosarcoma, rhabdomyosarcoma, or both. Other heterologous elements including angiosarcoma or osteoid have also been described rarely. ${ }^{1}$ Divergent rhabdomyosarcomatous differentiation has been reported previously in only seven cases of liposarcoma. ${ }^{1-5}$ All were dedifferentiated liposarcomas arising in the retroperitoneum and in all but one case rhabdomyosarcoma was found only in tumour recurrences. There is only a single previous case of rhabdomyosarcomatous differentiation in de novo liposarcoma, a retroperitoneal dedifferentiated tumour. ${ }^{5}$

Here, we describe a unique case of divergent rhabdomyosarcomatous differentiation in a de novo liposarcoma, which was of combined myxoid and pleomorphic subtypes arising in the thigh. Immunohistochemical and ultrastructural evidence is provided to support our diagnosis.

\section{Case report}

A 75 year old man presented with a six week history of a swelling in the right thigh. This was associated with oedema of the right leg, but there were no systemic symptoms. There was no evidence of any abdominal mass. On exploration, a mass was found to be displacing, but not invading, the femoral vessels and nerves. The tumour was easily mobilised except on the posterior surface where it was adherent to the pectineus muscle.

Macroscopically, the tumour consisted of a partly encapsulated fatty mass measuring 12 $\mathrm{cm}$ at its maximum dimension with a small amount of surrounding soft tissue. It was partly necrotic. Histologically, the tumour was a liposarcoma which was predominantly of myxoid type with a typical delicate branching capillary network and myxoid stroma (fig 1). In places, the tumour had more cellular pleomorphic areas. The latter were composed of spindle cells with notable nuclear pleomorphism and bizarre lipoblasts containing typical scalloped nuclei and cytoplasmic lipid vacuoles (fig 1, left upper inset). Mitotic activity was frequent $(21 / 10$ high power fields) within the pleomorphic areas and large foci of necrosis were seen. The tumour grade was 3 based on the method by Trojani et al. ${ }^{6}$

Cells within the pleomorphic areas had abundant eosinophilic cytoplasm and noticeably hyperchromatic nuclei (fig 1, right lower inset), many showing notable nuclear enlargement and irregular nuclear contours. Pleomorphic multinucleate tumour giant cells were also seen. No cross striations were visible by light microscopy. Immunohistochemistry was performed using the streptavidin-peroxidase complex technique with diaminobenzidine chromogen substrate. The pleomorphic cells showed intense cytoplasmic positivity using immunostains for desmin (diluted 1 in 25; Dako, High Wycombe, UK). Myoglobin (diluted 1 in 1000; Dako) and muscle specific actin (HHF 35) (diluted 1 in 40; Biomen, Finchampstead, UK) were also positive in these cells. A proportion of them were positive for fast myosin (diluted 1 in 600; Sigma, Poole, Dorset, UK) and sarcomeric actin (diluted 1 in 100; Sigma). No tumour cells stained for 